\title{
Truffles in the sky: the impact of stochastic and deterministic drivers on Rhizopogon communities of the Madrean Sky Island Archipelago
}

\author{
Carolina Piña Páez ${ }^{1}$, Adam Carson ${ }^{1}$, Daniel Luoma ${ }^{1}$, and Joseph Spatafora ${ }^{1}$ \\ ${ }^{1}$ Oregon State University
}

October 19, 2021

\begin{abstract}
The Madrean Sky Islands Archipelago (MSIA) comprises mountain "islands" whose Pine-Oak forests appear in stark contrast to the surrounding "sea" of Sonoran Desert vegetation. Rhizopogon (Boletales) consists of obligate ectomycorrhizal (EcM) symbionts that form truffle sporocarps and associate exclusively with Pinaceae. The objectives of this project were to describe the diversity of species of Rhizopogon across the MSIA and to characterize whether community structure is determined by host diversity, island identity, geographic distance, or some interaction among these factors. We selected nine islands, two sites were sampled per island: one site dominated by Pinus species and the other by Pseudotsuga menziesii var. glauca. Rhizopogon diversity was characterized from sporocarps and from bioassay-based EcM root tips derived from P. muricata, Ps. menziesii var. menziesii, and Ps. menziesii var. glauca seedlings inoculated with soil samples collected along transects established at each site. The ITS rDNA fungal barcode was amplified, and sequences were used in community analyses. Twenty-one $99 \%$ OTUs in the genus Rhizopogon were identified across nine sky islands. While differential host association with Pinus and Pseudotsuga is a significant driver of community composition, our results supported a stronger island effect. Furthermore, Rhizopogon communities associated within hosts are characterized by random phylogenetic structures across sky islands and are not structured by geographic distance. These results are consistent with a strong isolation effect involving historical habitat fragmentation of sky islands in response to past climate changes, and that both niche partitioning and stochastic demographic processes function in shaping Rhizopogon communities of the MSIA.
\end{abstract}

\section{Hosted file}

PinaPaez_etal_2.pdf available at https://authorea.com/users/441919/articles/542346-trufflesin-the-sky-the-impact-of-stochastic-and-deterministic-drivers-on-rhizopogon-communitiesof-the-madrean-sky-island-archipelago 